\title{
Time-domain RLS-based channel estimation for MIMO OFDM systems
}

\begin{abstract}
In this paper, an adaptive channel estimation scheme for MIMO OFDM systems based on time-domain training and recursive least squared (RLS) algorithm is proposed. Time orthogonal as well as simultaneously transmitted training sequences are considered. The channel is assumed to be slowly varying time-dispersive, i.e., constant during one OFDM symbol but changing from symbol to symbol. Channel estimation is performed in timedomain followed by zero-forcing equalization in the frequency-domain. The computational complexity is significantly reduced by applying the matrix inversion lemma. Simulation results show that the proposed estimator with time orthogonal training sequences has better estimation performance over a range of Doppler spreads compared to the case when the training sequences are simultaneously transmitted from the different transmit antennas.
\end{abstract}

Keyword: Channel estimation; MIMO; OFDM; RLS algorithm 